
OXIDO E 2,2-DITHIOBISPIRIDINA-N-ÓXIDO

\title{
MOLECULAR ABSORPTION SPECTROSCOPY IN THE UV-VIS REGION FOR THE QUINOLINE-N-OXIDE, NICOTINAMIDE-N-OXIDE AND 2,2-DITHIOBISPYRIDINE-N-OXIDE LIGANDS
}

LIMA, Francisco Jose Santos*; COSTA, Luiz Henrique Medeiros da; SILVA, Ademir Oliveira da, PEREIRA, Francisco Claudece.

Universidade Federal do Rio Grande do Norte, Centro de Ciências Exatas e da Terra Instituto de Química, Av. Senador Salgado Filho, s/n - Campus Universitário, Natal RN

${ }^{*}$ Autor correspondente

limafjs@yahoo.com

Received 06 June 2017; received in revised form 30 November 2017; accepted 14 December 2017

\section{RESUMO}

A proposta deste trabalho foi analisar e quantificar através de parâmetros espectrais, as bandas de absorção, especificamente na região uv-visível de alguns ligantes $\mathrm{N}$-óxidos. A partir dos espectros, foram calculadas para cada um deles, a área sob a banda de absorção, a absortividade molar, o coeficiente de absorção integrado e a força do oscilador pelo método da aproximação pela Gaussiana, usando a avaliação proposta por Drago e Figgs. De acordo com os resultados obtidos, pode-se concluir que os compostos heterocíclicos estudados, absorvem muito bem radiação eletromagnética na região do ultravioleta. Para os ligantes QUI-NO e NIC-NO foi observado que há dois tipos de bandas, primárias e secundárias, sendo transições do tipo $\pi \rightarrow \pi^{*}$ e $n \rightarrow \pi^{*}$, respectivamente. Para a DITBIS-NO, houve apenas a ocorrência de uma banda primária (transição 1) e dois ombros adicionais, sendo esta apresentando o maior coeficiente de absortividade observado. Estes ligantes são esperados como potenciais doadores de elétrons em sistemas complexos, que têm como meta, alterar os níveis de energia do sistema eletrônico a fim de promover um melhor aproveitamento da energia absorvida e, portanto uma melhor eficiência.

Palavras-chave: Espectroscopia uv-vis, ligantes $\mathrm{N}$-óxidos, força do oscilador.

\section{ABSTRACT}

Required The purpose of this study was to analyze and quantify spectral parameters, the absorption bands, specifically in the uv-visible region of some $\mathrm{N}$-oxide ligands. From the spectra, the area under the absorption band, the molar absorptivity, the integrated absorption coefficient and the oscillator strength by the Gaussian approximation method were calculated for each of them, using the proposed evaluation by Drago and Figgs. According to the obtained results, it can be concluded that the heterocyclic compounds studied absorb very well electromagnetic radiation in the ultraviolet region. For the ligands QUI-NO and NIC-NO it was observed that there are two types of bands, primary and secondary, being transitions of type $\pi \rightarrow \pi{ }^{*}$ and $n \rightarrow$ $\pi^{*}$, respectively. For DITBIS-NO, there was only one primary band (transition 1 ) and two additional shoulders, which had the highest observed absorbance coefficient. These ligands are expected as potential electron donors in complex systems whose purpose is to change the energy levels of the electronic system in order to promote a better utilization of the absorbed energy and therefore a better efficiency.

Keywords: UV-VIS spectroscopy, N-oxide ligand, oscillator strength. 


\section{INTRODUÇÃO:}

A espectroscopia de absorção molecular, na região do UV-Visível, é uma técnica bastante utilizada para estudos da interação da radiação eletromagnética com moléculas, átomos e íons. A absorção desta radiação promove elétrons de um estado relativo de baixa energia (HOMO) para outro estado de mais alta energia (LUMO). Estudos mostram, fundamentados em regras de seleção, que em sistemas moleculares, há transições do tipo permitidas e proibidas (SOLOMONS, 1990; SILVERSTEIN et al., 1994; PAVIA, et al., 2010). A primeira atribuída às transições $\pi \rightarrow \pi^{*}$, enquanto que a segunda, transições $n \rightarrow \pi^{*}$. Esses tipos de transições geralmente são observados em moléculas heterocíclicas, tais como presentes nas moléculas de piridina, quinolina, isoquinolina entre outras. (SILVERSTEIN et al., 1994; PAVIA, et al., 2010; MARTINHO, 1994; ASSIS, 2013).

De acordo com Drago (DRAGO, 1965), Figgs (FIGGS 1966) e estudos mais recentes (LIMA et al, 1996), os espectros eletrônicos das moléculas, permitem avaliar algumas propriedades espectrais e é possível calcular a força do oscilador, que tem como objetivo quantificar a intensidade de uma transição, ao se comparar a probabilidade de uma transição experimental em relação à probabilidade de uma transição teórica, a partir dos espectros de absorção das amostras em solução ou no estado sólido.

Segundo Pedrosa (2004), avaliar as propriedades espectroscópicas e os parâmetros óptico-eletrônicos de complexos contendo íons lantanídeos, tem sido largamente investigado na literatura devido às diversas possibilidades de aplicações, como por exemplo, em lasers, sensores luminescentes para espécies químicas, células fotoquímicas, entre outras. (PEDROSA et. al, 2004)

O objetivo deste trabalho foi analisar as bandas de absorção na região uv-visível de alguns ligantes $\mathrm{N}$-óxido, dando continuidade a um trabalho anterior, (LIMA et al., 2016), no qual foram avaliados os parâmetros de reatividades moleculares, obtidas medidas de condutância molar, e correlacionados os parâmetros de reatividades com 0 comportamento condutimétrico destes ligantes. A partir dos espectros neste trabalho, foi quantificada a área sob a banda de absorção, a absortividade molar $\varepsilon_{\text {máx }}$ o coeficiente de absorção integrado $\int \varepsilon(\sigma) d \sigma$, e a força do oscilador $f$, pelo método da Gaussiana, também conhecido pelo método da meia-banda, usando a avaliação proposta por DRAGO e FIGGS (DRAGO, 1965; FIGGIS, 1966).

\section{METODOLOGIA:}

Foram preparadas soluções aquosas dos compostos: Quinolina-N-Óxido $\left(\mathrm{C}_{9} \mathrm{H}_{7} \mathrm{NO}-\mathrm{QUI}\right.$ $\mathrm{NO})$, Nicotinamida-N-Óxido $\left(\mathrm{C}_{6} \mathrm{H}_{6} \mathrm{~N}_{2} \mathrm{O}_{2}\right.$ - NIC-NO) e a 2,2-Dithiobispiridina-N-Oxido $\left(\mathrm{C}_{10} \mathrm{H}_{8} \mathrm{~N}_{2} \mathrm{O}_{2} \mathrm{~S}_{2}\right.$ DITBIS-NO), de concentração $1 \times 10^{-6} \mathrm{~mol} \mathrm{~L}^{-1}$, e registrado seus espectros na região uv-visível em cubeta de quartzo de $1,00 \mathrm{~cm}$ de caminho óptico. A Figura 01 mostra a modelagem para as moléculas estudadas.

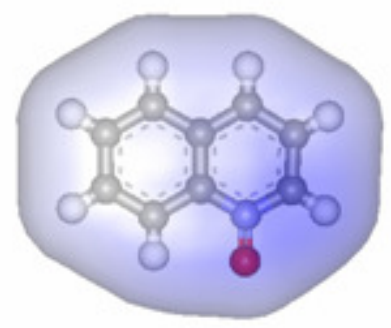

Quinolina-N-óxido

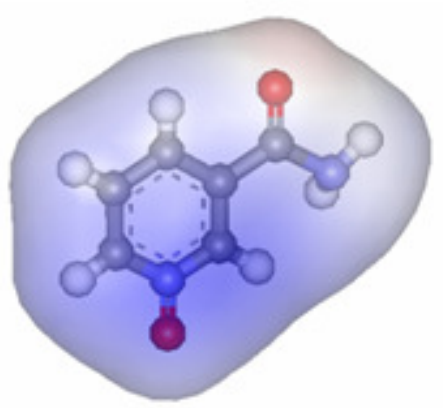

Nicotinamida-N-óxido

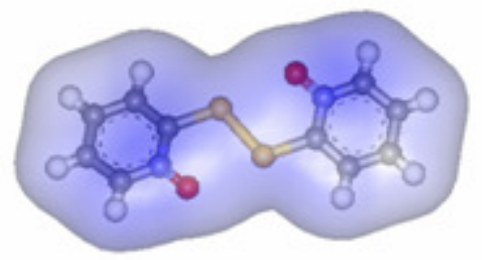

2,2-Dithio bispiridina- $N$-óxido

Figura 01 - Modelagem das moléculas estudadas. (LIMA et. al, 2016) 
Para a visualização do espectro, foi realizada uma varredura no intervalo de comprimento de onda de 190 a $800 \mathrm{~nm}$, através de um equipamento UV-VIS Spectrophotometer SHIMADZU 1650PC, duplo-feixe, com precisão de absorbância de $\pm 0,004$ e precisão de comprimento de onda de $\pm 0,3 \mathrm{~nm}$, que permite varreduras de $190-800 \mathrm{~nm}$. As transições relevantes ocorreram entre 200-400 nm. A temperatura registrada do experimento foi de 20 ${ }^{\circ} \mathrm{C}$.

A partir do espectro, neste trabalho foi calculada a área sob a banda de absorção, a absortividade molar, o coeficiente de absorção integrado e a força do oscilador (LIMA et al., 1996; LIMA et al., 2014; ZHAO et al., 2016), pelo método da Gaussiana, também conhecido como método da meia-banda, segundo DRAGO e FIGGS (DRAGO, 1965; FIGGIS, 1966).

$$
\begin{array}{ll}
\text { DRAGO: } f=4,6 \times 10^{-9} \int \varepsilon_{(\sigma)} \mathrm{d} \sigma & \text { Eq. } 01 \\
\text { FIGGS: } f=4,32 \times 10^{-9} \int \varepsilon_{(\sigma)} \mathrm{d} \sigma & \text { Eq. } 02
\end{array}
$$

Nos métodos de Drago e Figgs há uma diferença na constante que precede a integral, que tem como propósito corrigir para mais e/ou para menos, a estimativa da área sob uma banda de absorção, que envolve uma transição eletrônica. A finalidade disto é corrigir a avaliação da área sob a curva de absorção pelo método da meia-banda $\int \varepsilon(\sigma) d \sigma \cong \varepsilon_{\max } \Delta \sigma_{1 / 2}$, também conhecido como método de aproximação pela gaussiana (DRAGO, 1965; FIGGIS, 1966). Estes valores envolvem constantes fundamentais que quantificam a transição "ab initio" de um elétron no átomo de hidrogênio entre os estados fundamental e primeiro estado excitado e visam quantificar uma transição teórica, como referência, para estimar a probabilidade de uma transição experimental, denominada de força do oscilador (ATKINS, 1983; ATKINS, 1991; LIMA et al., 1996; ZHAO et al., 2016). A integral $\int \varepsilon(\sigma) d \sigma$ se refere a área sob uma curva de absorção, que neste caso é obtida em termos da absortividade molar $\varepsilon\left(\mathrm{L} \mathrm{mol}^{-1} \mathrm{~cm}^{-1}\right)$ versus $\sigma=1 / \lambda\left(\mathrm{cm}^{-1}\right)$, e possui unidades de $\mathrm{L} \mathrm{mol}^{-1} \mathrm{~cm}^{-2}$.

\section{RESULTADOS:}

A Figura 02 mostra os espectros de absorção dos ligantes selecionados e a Tabela 01 mostra os parâmetros calculados neste trabalho

\section{DISCUSSÕES}

Segundo Pavia e co-autores (PAVIA, et al., 2010), e Santos (SANTOS, 2008), ambos atribuíram à compostos aromático, bandas primárias, denominada de transições permitidas, que são bandas mais expressivas que ocorrem em regiões de maiores energias, e bandas secundárias, denominada de transição proibidas em regiões de menores energias. Assim, as bandas primárias possuem um coeficiente de absortividade maior que os das bandas secundárias devido a sua faixa espectral, mas também devido as suas absorbâncias apresentarem maiores intensidades.

De acordo com os resultados obtidos, observaram-se bandas primárias e secundárias para as moléculas QUI-NO e NIC-NO. Já na molécula DITBIS-NO identificou-se apenas bandas primárias, porém há formação de dois ombros até $330 \mathrm{~nm}$. Os espectros estão mostrados na Figuras 01. Para a Quinolina-Nóxido há duas bandas de absorção. A banda primária encontra-se na região entre 220,5 $236,5 \mathrm{~nm}$, sendo a transição $1\left(\pi \rightarrow \pi^{\star}\right)$, que possui um coeficiente de absortividade da ordem de $9,523 \times 10^{5}$. Na região entre $290-340,5 \mathrm{~nm}$ observa-se a transição $2\left(n \rightarrow \pi^{\star}\right)$, que possui um menor coeficiente de absortividade que a transição 1, sendo da ordem de $1,540 \times 10^{5}$, mostrado na Tabela 01 . No espectro da molécula NIC-NO verificou-se também a presença de duas bandas, semelhante ao que ocorreu na molécula QNO-NO. A transição 1 está localizada na região entre 200 - 228,5 nm, sendo uma transição do tipo $\pi \rightarrow \pi$ do qual possui um coeficiente de absortividade de $5,724 \times 10^{5}$. Já a transição 2 ocorreu entre 239 - 273,5 nm, sendo esta considerada uma transição do tipo $n \rightarrow \pi^{\star}$, tendo um coeficiente de absortividade de $2,812 \times 10^{5}$, mostrado na Tabela 01. Por último, o ligante DITBIS-NO ocorreu apenas uma banda com uma transição bem resolvida e dois ombros adjacentes. Nessa banda, observou-se o pico de sua ocorrência numa região de 217,5 - 249 nm da qual possui um coeficiente de absortividade da ordem de $10,562 \times 10^{5}$ (transição 1), conforme mostrado na Tabela 01. Assim, sendo, foi atribuída a uma transição composta que apresenta dois tipos, sendo $\pi \rightarrow \pi^{*}$ e $n \rightarrow \pi^{*}$. 


\section{CONCLUSÃO}

De acordo com os resultados, conclui-se que os compostos estudados absorvem na região do ultravioleta, não apresentando absorções significativas na região do visível, sendo por isso incolores em solução aquosa. Para os ligantes QUI-NO e NIC-NO há dois tipos de bandas: primárias e secundárias, sendo transições do tipo $\pi \rightarrow \pi^{*}$ e $n \rightarrow \pi^{*}$, respectivamente. Percebeu-se, também que as bandas primárias possuem coeficiente de absortividade e força do oscilador maior que as bandas secundárias, apesar de possuírem maiores energias de excitação. Esta observação, para estes compostos, é importante, pois implica que a probabilidade destas transições não necessariamente depende apenas da energia e sim principalmente do mecanismo de cada tipo de transição. Para a DITBIS-NO, há apenas a ocorrência de uma transição mais intensa, composta com dois ombros.

Observando 0 coeficiente de absortividade molar e a força do oscilador, notase que quanto maior este coeficiente, maior o valor para esse parâmetro. Dentre os ligantes que foram estudados, a DITBIS-NO foi a que revelou maiores resultados, indicando que possui relevante capacidade de absorção de fótons nesta região, podendo ser um sistema interessante para captação de radiação em sistemas fotoemissores, dependendo apenas dos mecanismos de transferência radiativa e nãoradiativa com outros sistemas acoplados. Outros estudos estão em desenvolvimento para a avaliação do momento de dipolo de transição e estimativas do tempo de transição radiativa e não-radiativa, que possibilitem a avaliação de rendimentos quânticos em sistemas fotoindutores.

\section{AGRADECIMENTOS}

Os autores são muito gratos ao $\mathrm{PIBIC/PIBIT/PROPESQ/UFRN/CNPq,} \mathrm{pelo}$ incentivo à pesquisa em iniciação científica e inovação tecnológica, especialmente direcionada à química fundamental.

\section{REFERÊNCIAS}

1 - ASSIS, O. B. G., 2013 - A Asa da Borboleta e a Nanotecnologia: Cor Estrutural - Rev. Bras. Ensino Fís. 35(2), 2301(1)-2301(9), São Paulo.

2 - ATKINS, P. W., 1983 - Molecular Quantum
Mechanics - 2a Ed., Oxford University

Press - Printed in Great Britain.

3 - ATKINS, P. W., 1991 - Quanta - 2a Ed.

Oxford University Press, New York.

4 - DRAGO, R. S., - Physical Methods in Inorganic Chemistry, $1965-$ Vau Nostrand Reinhold Company , printed Holland;

5 - FIGGIS, B. N., Introduction To Ligands Fields, 1966 - Interscience Publishers, New York, Copyright by John Wiley \& Sons, Inc.;

6 - LIMA, F. J. S., BRITO, H. F., SILVA, A. G. SILVA, A. O. BRAGA, C. C. M, LIMA, A. J. P., CARDOSO, M. C. C., 1996 - O Uso da Força do Oscilador na Avaliação de Intensidades Espectrais - Anais da Associação Brasileira de Química, 45 (1), 31-35.

7 - LIMA, F. J. S.; COSTA, L. H. M.; SILVA, A. O., 2014 - Estudos Espectroquímicos do Íon $\mathrm{UO}_{2}{ }^{2+}$ Coordenado no Acetato de Uranila $\mathrm{UO}_{2}\left(\mathrm{H}_{3} \mathrm{CCOO}\right)_{2} \cdot 2 \mathrm{H}_{2} \mathrm{O}$, , Periódico Tchê Química, Vol. 11, (22), 33-45.

8 - LIMA, F. J. S.; COSTA, L. H. M.; AZEVEDO, D. M., SILVA, A. O. e PEREIRA, F. C., 2016 - Parâmetros de Reatividade Molecular e a Correlação Com a Condutividade Molar da Quinolina-NÓxido, Nicotinamida-N-Óxido e a 2,2Dithiobispiridina-N-Óxido, Periódico Tchê Química, Vol. 13 (26), 88-96.

9 - MARTINHO, J. M. G., 1994 - Espectroscopia de Absorção no Ultravioleta e Visível, QUÍMICA, 52, 44.

10 - PAVIA, D. L.; LAMPMAN, G. M.; KRIZ, G. S. e VYVYAN, J.R.; 2010 - Introdução à Espectroscopia; São Paulo: 4. Ed. Norteamericana.

11 - Pedrosa, A. M. G., PIMENTEL, P. M., BATISTA M. K. S., LIMA F. J. S. E MELO D. M. A., 2004 - Propriedades Espectroscópicas e Parâmetros ÓpticosEletrônicos dos Complexos $\mathrm{Ln}\left(\mathrm{NO}_{3}\right)_{3} .2 \mathrm{DTPO} .4 \mathrm{H}_{2} \mathrm{O}(\mathrm{Ln}=\mathrm{Nd}$ e $\mathrm{Er})$, Química Nova, Vol. 27, №. 3, 367-370.

12 - SANTOS A. F. S., 2008 - Determinação de Parâmetros Ópticos de Materiais por Análise de Kramers-Kronig de Espectros de Infravermelho, 2008. Dissertação (Mestrado em Ciência dos Materiais) - 
Universidade Estadual Paulista Júlio de Mesquita Filho, São Paulo - Ilha Solteira.

13 - SOLOMONS, T. W. G., 1990

Fundamentals of Organic Chemistry - $3^{a}$

Ed. Jonh Wiley Sons, printed in the U.S.A.

14 - SILVERSTEIN, R. M., BASSLLER, G. C.

AND MORRILL, T. C. 1994, Identificação

Espectrométrica de Compostos

Orgânicos, - 5a. Ed. Guanabara Koogan

S.A

15 - ZHAO, H., CHANG, J., DU, L., 2016 - Effect

Of Hydrogen Bonding On The

Spectroscopic Properties Of Molecular

Complexes With Aromatic Rings As

Acceptors, Comp. Theor. Chem., 1084, $126-132$ 

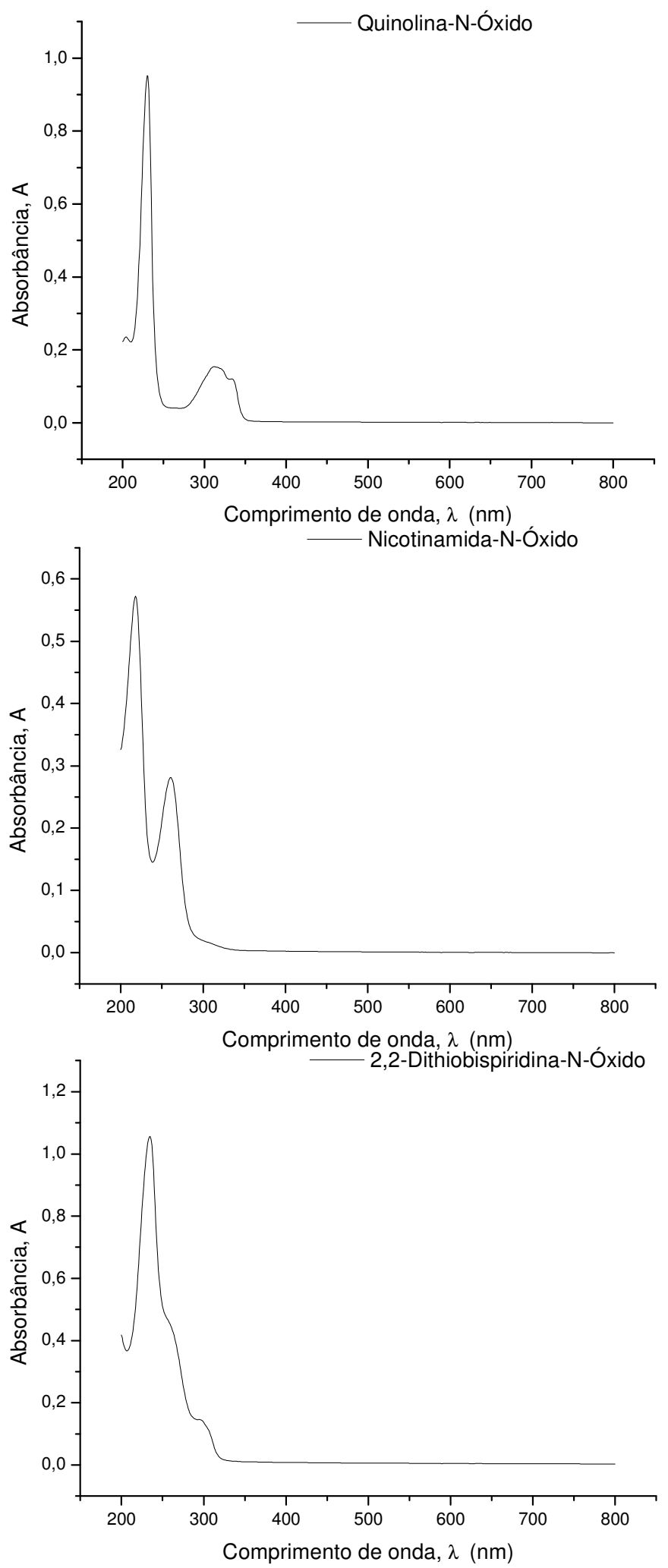

Figura 02 - Varredura do espectro de absorção na região uv-visível completo dos ligantes N-óxidos. Quinolina-N-Óxido (QUI-NO); Nicotinamida-N-Óxido (NIC-NO) e a 2,2-Dithiobispiridina-N-Óxido (DITBIS-NO) em solução aquosa $1,00 \times 10^{-6} \mathrm{~mol} \mathrm{~L}^{-1}$. 
Tabela 01 - Intervalo espectral, área pelo método da Gaussiana e Força do Oscilador pelo método de Drago e Figgs para as soluções aquosas dos ligantes de concentração $1 \times 10^{-6} \mathrm{~mol} \mathrm{~L}^{-1}$.

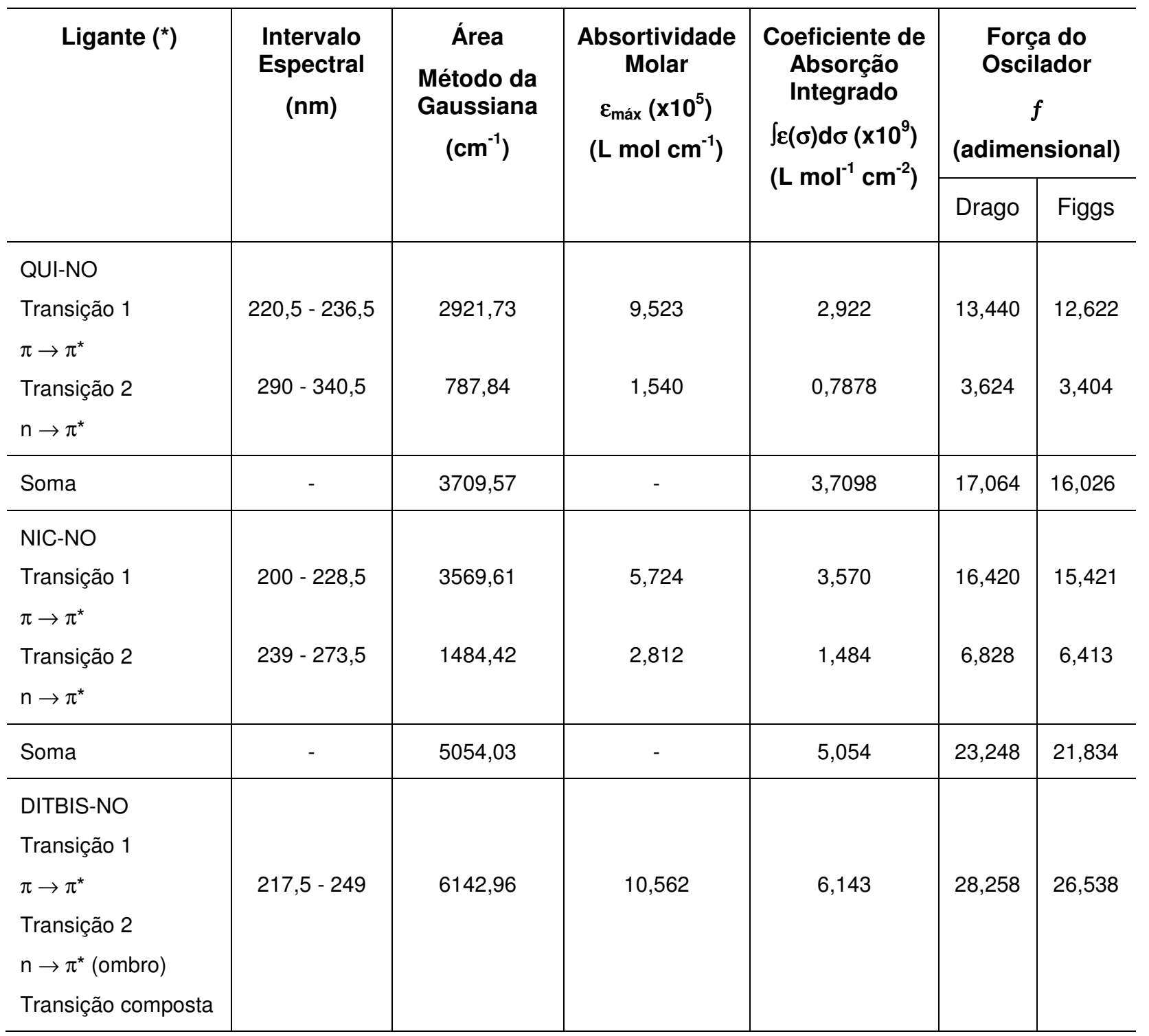

$\left(^{*}\right)$ - Quinolina-N-Óxido (QUI-NO); Nicotinamida-N-Óxido (NIC-NO) e 2,2-Dithiobispiridina-N-

Óxido (DITBIS-NO) em solução aquosa de concentração $1,00 \times 10^{-6} \mathrm{~mol} \mathrm{~L}^{-1}$.

PERIÓDICO TCHÊ QUÍMICA • www.periodico.tchequimica.com • Vol. 15 N. 29

- ISSN 1806-0374 (impresso) • ISSN 1806-9827 (CD-ROM) • ISSN 2179-0302 (meio eletrônico)

(C) 2018. Porto Alegre, RS. Brasil

The Periódico Tchê Química (ISSN: 1806-0374; 2179-0302) is an open-access journal since 2004. Journal DOI: 10.52571/PTQ. http://www.tchequimica.com. This text was introduced in this file in 2021 for compliance reasons.

(๑) The Author(s)

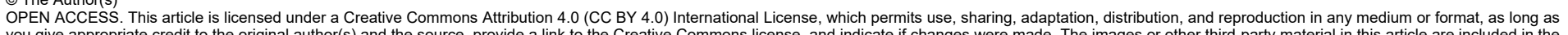

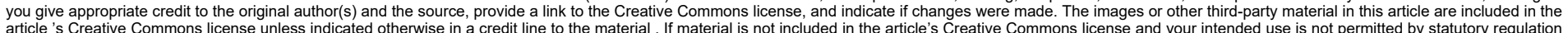
article 's Creative Commons license unless indicated otherwise in a credit line to the material . If material is not included in the article's Creative Commons license and your intended use is not permitted by statutory regulation or exceeds the permitted use, you will need to obtain permission directly from the copyright holder. To view a copy of this license, visit http://creativecommons.org/licenses/by/4.0/. 